\title{
A UNIFIED APPROACH FOR THE ESTIMATION OF INSTANTANEOUS FREQUENCY AND ITS DERIVATIVES FOR NON-STATIONARY SIGNALS ANALYSIS
}

\author{
*Irena Orović, "Andjela Draganić, "Srdjan Stanković and ${ }^{* *}$ Ervin Sejdić \\ *Faculty of Electrical Engineering, University of Montenegro, Dzordza Vasingtona bb, 81000 \\ Podgorica, Montenegro \\ ** Department of Electrical and Computer Engineering, Swanson School of Engineering, \\ University of Pittsburgh, Pittsburgh, PA, 15261, USA
}

\begin{abstract}
A unified approach for the estimation of the first three phase derivatives of non-stationary signals is proposed in this paper. The possibility to accurately estimate phase derivatives is important in many applications dealing with objects velocity, acceleration and acceleration rate, such as the radar applications and mechanics. The estimation approach is based on definition of the complex-lag distribution. The proposed distribution is inspired by the concepts of complex analysis theory. The general form of distribution for the estimation of the first, second and third derivative of the phase is derived from the specific individual cases. The theoretical considerations are illustrated in the example with fast varying signal phase function.
\end{abstract}

\section{INTRODUCTION}

During the last two decades, time-frequency representations have been used for the analysis of highly non-stationary signals such as biomedical signals, radar and sonar signals, signals in communication, etc. Since there is no ideal time-frequency distribution that can be used for all signals, various linear (spectrogram), quadratic (Wigner distribution, distributions from the Cohen's class, the S-method) and the higher order distributions (e.g., polynomial and complex-lag distributions) time-frequency distributions have been introduced [1]-[12]. A desirable distribution property is good concentration in the time-frequency domain.

Time-frequency distributions with a complex-lag argument are used for an efficient analysis of signals with fast varying phase functions and instantaneous frequencies [9]. However, due to the fast phase variations most of the mentioned distributions do not provide satisfactory concentration, causing errors when estimating instantaneous frequency or higher phase derivatives. Similar constraints hold when estimating the second ([8]) or higher order phase derivatives. Hence, in the sequel we consider an approach that can provide a unified analysis and accurate estimation for the first three phase derivatives.

Unlike the standard higher order complex-lag distributions, the proposed approach is based on the complex-lag signal moment composed of only two signal terms. The proposed distribution is based on the principles from the complex analysis theory [5]. Namely, the analogy with the complex analysis theory has been used to define general and specific distribution forms for the estimation of the first few phase derivatives. This method is applied to monocomponent signals. In the case of multicomponent signals, cross-terms appear due to nonlinearity of the considered time-frequency distributions. Hence, the components should be separated prior to applying this approach to multicomponent signals.

The paper is organized as follows. The theoretical background on the complex analysis and Cauchy integral formula are given in Section 2. The specific forms of distribution for the estimation of the first, second and third order derivative of the phase are derived in Section 3, while the general distribution form, as well as its Lform are given in Section 4. The experimental evaluation is done in Section V. The concluding remarks are given in Section VI.

\section{THEORETICAL BACKGROUND}

According to the complex analysis theory, a holomorphic function $f(z)$, defined on the closed disc $D=\left\{z:\left|z-z_{0}\right| \leq r\right\}$, can be completely determined by using the values on the boundary circle $C$ of the disc $D$ : 


$$
f(t)=\frac{1}{2 \pi j} \oint \frac{f(z)}{z-t} d z .
$$

An arbitrary derivative of function $f(z)$ can be obtained as follows:

$$
f^{K}(t)=\frac{K !}{2 \pi j} \oint \frac{f(z)}{(z-t)^{K+1}} d z .
$$

In order to make a certain analogy between the complex functions and signals that we use in real applications, instead of function $f(z)$, we observe the phase of the signal $x(t)=r e^{j \varphi(t)}$. If it is assumed that the boundary circle $C$ is centered at instant $t$ and if $z=t+\tau e^{j \theta}$ holds $(\tau$ is the radius and $\theta \epsilon[0,2 \pi]$ ), then an arbitrary $K$-th phase derivative can be defined as [11]:

$$
\varphi^{(K)}(t)=\frac{K !}{2 \pi \tau^{K}} \int_{0}^{2 \pi} \varphi\left(t+\tau e^{j \theta}\right) e^{-j k \theta} d \theta .
$$

In the sequel, we will observe some specific phase derivatives to provide appropriate discrete expressions, which will further lead to the definition of the corresponding distributions.

\section{ESTIMATION OF PHASE DERIVATIVES}

\subsection{The first derivative of the phase - instantaneous frequency}

According to (3), $K=1$ is used when observing the first derivative of the phase:

$$
\varphi^{(1)}(t)=\frac{1}{2 \pi \tau} \int_{0}^{2 \pi} \varphi\left(t+\tau e^{j \theta}\right) e^{-j \theta} d \theta .
$$

The discretization of the previous relation based on $N$ discrete points will result in:

$$
\varphi^{(1)}(t) \tau=\sum_{k=0}^{N-1} \varphi\left(t+\frac{\tau}{N} e^{j 2 \pi k / N}\right) e^{-j 2 \pi k / N} .
$$

The right hand side represents the phase of the complex-lag signal moment $M(t, \tau)$ that can be defined as follows:

$$
M(t, \tau)=\prod_{k=0}^{N-1} x^{-\frac{j 2 \pi k}{N}}\left(t+\frac{\tau}{N} e^{\frac{j 2 \pi k}{N}}\right) .
$$

By applying the Fourier transform to the moment $M(t, \tau)$, the $N$-th order complex-lag distribution for the instantaneous frequency estimation is obtained. In the following analysis we focus on the simplest case based on the two-term moment function. Hence, only two discretization points on the unit circle are considered: $(a+j b)$ and $-(a+j b)$. The complex-lag moment function can be now written as:

$$
M(t, \tau)=\prod_{i=-1}^{1} x^{\operatorname{sign}(i)(a+j b)}\left(t+\frac{\tau}{2 \operatorname{sign}(i)(a+j b)}\right) .
$$

The corresponding complex-lag distribution for the instantaneous frequency estimation is obtained as the Fourier transform of the moment function:

$$
\begin{aligned}
& C T D_{N=2}^{I}(t, \omega)=\int_{-\infty}^{\infty} M(t, \tau) e^{-j \omega \tau} d \tau= \\
& =\int_{-\infty}^{\infty} \prod_{i=-1}^{1} x^{\operatorname{sign}(i)(a+j b)}\left(t+\frac{\tau}{2 \operatorname{sign}(i)(a+j b)}\right) e^{-j \omega \tau} d \tau .
\end{aligned}
$$

Applying the Taylor series expansion to the phase of the moment function $M(t, \tau)$, we get:

$$
\begin{aligned}
& \varphi_{M}(t, \tau)=\varphi^{(1)}(t) \tau+\varphi^{(3)}(t) \frac{\tau^{3}}{2^{2} 3 !(a+j b)^{2}}+ \\
& +\varphi^{(5)}(t) \frac{\tau^{5}}{2^{4} 5 !(a+j b)^{4}}+\varphi^{(7)}(t) \frac{\tau^{7}}{2^{6} 7 !(a+j b)^{6}}+\ldots
\end{aligned}
$$

The higher phase derivatives represent the spreading factor that causes the distribution spread along the instantaneous frequency. An appropriate choice of discretization points can significantly reduce the spreading factor. In that sense, let us observe two specific points: $\sqrt{j}$ and $-\sqrt{j}$. In this case the distribution (8) can be rewritten in the form:

$$
\begin{aligned}
& \operatorname{CTD}_{N=2}^{I}(t, \omega)= \\
& =\int_{-\infty}^{\infty} \prod_{i=-1}^{1} x^{\operatorname{sign}(i) \sqrt{j}}\left(t+\frac{\tau}{2 \operatorname{sign}(i) \sqrt{j}}\right) e^{-j \omega \tau} d \tau .
\end{aligned}
$$

The moment phase function given by (9) becomes:

$$
\begin{aligned}
& \varphi_{M}(t, \tau)=\varphi^{(1)}(t) \tau-j \varphi^{(3)}(t) \frac{\tau^{3}}{2^{2} 3 !}- \\
& -\varphi^{(5)}(t) \frac{\tau^{5}}{2^{4} 5 !}+j \varphi^{(7)}(t) \frac{\tau^{7}}{2^{6} 7 !}+\ldots
\end{aligned}
$$

Note that the terms of order $4 n-1(n=1,2, \ldots)$ contain imaginary unit $j$. Thus, their influence can be eliminated by introducing the modification:

$$
M^{a}(t, \tau)=e^{\text {jangle }(M(t, \tau))} .
$$


The corresponding distribution is defined as the Fourier transform of $M^{a}(t, \tau)$ :

$$
C T D_{N=2}^{I}(t, \omega)=\int_{-\infty}^{\infty} M^{a}(t, \tau) e^{-j \omega \tau} d \tau
$$

\subsection{The second derivative of the phase}

The second derivative of the phase has been known in the applications as the instantaneous frequency rate. By using $K=2$ in (3), and performing the discretization in $N$ points, the following expression is derived:

$$
\frac{\varphi^{(2)}(t) \tau^{2}}{2 !}=\sum_{k=0}^{N-1} \varphi\left(t+\frac{\tau}{N} e^{j 2 \pi k / N}\right) e^{2\left(-\frac{j 2 \pi k}{N}\right)} .
$$

In order to provide the second derivative of the phase estimation, the moment function is defined as:

$$
M(t, \sqrt{\tau})=\prod_{k=0}^{N-1} x^{2\left(-\frac{j 2 \pi k}{N}\right)}\left(t+\sqrt{\frac{2 ! \tau}{N}} e^{\frac{j 2 \pi k}{N}}\right) .
$$

The above relation is a counterpart of (6) for the second order derivative. Again, the corresponding distribution is obtained as the Fourier transform of the moment function:

$$
C T D^{I I}(t, \omega)=\int_{0}^{\infty} M(t, \sqrt{\tau}) e^{-j \omega \tau} d \tau=\int_{0}^{\infty} M(t, \tau) e^{-j \omega \tau^{2}} d \tau
$$

The simplest case is based on the two-terms moment function (i.e. two discretization points $\pm(a+\mathrm{j} b)$ ):

$$
\begin{aligned}
& C T D_{N=2}^{I I}(t, \omega)= \\
& =\int_{0}^{\infty} \prod_{i=-1}^{1} x^{(a+j b)^{2}}\left(t+\frac{\tau}{\operatorname{sign}(i)(a+j b)}\right) e^{-j \omega \tau^{2}} d \tau .
\end{aligned}
$$

The general form of the moment phase function is obtained by using the Taylor series expansion as follows:

$$
\begin{aligned}
& \varphi_{M}(t, \tau)=2(a+j b)^{2} \varphi(t)+\varphi^{(2)}(t) \tau^{2}+ \\
& 2 \varphi^{(4)}(t) \frac{\tau^{4}}{4 !(a+j b)^{2}}+2 \varphi^{(6)}(t) \frac{\tau^{6}}{6 !(a+j b)^{4}} \ldots
\end{aligned}
$$

Note that, as in the case of the instantaneous frequency estimation, the spreading factor contains the phase derivatives different from the one we want to estimate. The spreading factor depends on the choice of parameters $a$ and $b$. Following the analogy with the first phase derivative, the points $\pm \sqrt{j}$ are observed. The distribution (16) for these specific points becomes:

$$
C T D_{N=2}^{I I}(t, \omega)=\int_{0}^{\infty} x^{j}\left(t+\frac{\tau}{\sqrt{j}}\right) x^{j}\left(t-\frac{\tau}{\sqrt{j}}\right) e^{-j \omega \tau^{2}} d \tau
$$

The moment phase function contains the following terms:

$$
\begin{aligned}
& \varphi_{M}(t, \tau)=2 j \varphi(t)+\varphi^{(2)}(t) \tau^{2}-2 j \varphi^{(4)}(t) \frac{\tau^{4}}{4 !}- \\
& -2 \varphi^{(6)}(t) \frac{\tau^{6}}{6 !}+2 j \varphi^{(8)}(t) \frac{\tau^{8}}{8 !}+2 \varphi^{(10)}(t) \frac{\tau^{10}}{10 !}+\ldots
\end{aligned}
$$

The spread factor is reduced by eliminating imaginary terms, which will improve the concentration along the second derivative of the phase and consequently, the estimation precision. Hence, instead of the moment function $M(t, \tau)=e^{j \varphi_{M}(t, \tau)}$, the modified moment definition $M^{a}(t, \tau)=e^{\text {jangle }(M(t, \tau))}$ is used. In this way, we eliminate the influence of phase derivatives whose order is $4 n(n=1,2, \ldots)$.

\subsection{The third derivative of the phase}

The same concepts, employed in the case of the first and the second derivative of the phase, can be used for the estimation of the third derivative of the phase. The general two-term signal moment adapted to the third derivative of the phase estimation is defined by:

$$
M(t, \tau)=\prod_{i=-1,}^{1} x^{\operatorname{sign}(i)(a+j b)^{3}}\left(t+\frac{\sqrt[3]{3} \tau}{\operatorname{sign}(i)(a+j b)}\right),
$$

while the corresponding distribution is defined as:

$$
\begin{aligned}
& C T D_{N=2}^{I I I}(t, \omega)= \\
& =\int_{-\infty}^{\infty} \prod_{i=-1}^{1} x^{\operatorname{sign}(i)(a+j b)^{3}}\left(t+\frac{\sqrt[3]{3} \tau}{\operatorname{sign}(i)(a+j b)}\right) e^{-j \omega \tau^{3}} d \tau .
\end{aligned}
$$

The general form of the moment phase function for two arbitrary discretization points $(a+j b)$ and $(-a-j b)$ on the unit circle is given by:

$$
\begin{aligned}
& \varphi_{M}(t, \tau)=2 \varphi^{(1)}(t) \sqrt[3]{3} \tau(a+j b)^{2}+\varphi^{(3)}(t) \tau^{3}+2 \varphi^{(5)}(t) \frac{\sqrt[3]{3^{5}} \tau^{5}}{5 !(a+j b)^{2}}+ \\
& 2 \varphi^{(7)}(t) \frac{\sqrt[3]{3^{7}} \tau^{7}}{7 !(a+j b)^{4}}+2 \varphi^{(9)}(t) \frac{\sqrt[3]{3^{9}} \tau^{9}}{9 !(a+j b)^{6}}+2 \varphi^{(1)}(t) \frac{\sqrt[3]{3^{11}} \tau^{11}}{11 !(a+j b)^{8}}+\ldots
\end{aligned}
$$

Here, by an appropriate selection of parameters $a$ and $b$, we aim to eliminate the neighboring derivatives $\varphi^{(1)}(t)$ and $\varphi^{(5)}(t)$, which make the highest influence in terms 
of concentration spread. Hence, we observe the set of equations in the form:

$$
\begin{aligned}
& (a+j b)^{2}=j A, \\
& (a+j b)^{-2}=j B
\end{aligned}
$$

where $A$ and $B$ are constants. It can be easily shown that $a+j b=\sqrt{j}$ and its symmetrical point on the unit circle, satisfy both constraints. The phase of the moment function is thus given by:

$$
\begin{aligned}
& \varphi_{M}(t, \tau)=j 2 \varphi^{(1)}(t) \sqrt[3]{3} \tau+\varphi^{(3)}(t) \tau^{3}-j 2 \varphi^{(5)}(t) \frac{\sqrt[3]{3^{5}} \tau^{5}}{5 !} \\
& -2 \varphi^{(7)}(t) \frac{\sqrt[3]{3^{7}} \tau^{7}}{7 !}+j 2 \varphi^{(9)}(t) \frac{\sqrt[3]{3^{9}} \tau^{9}}{9 !}+2 \varphi^{(11)}(t) \frac{\sqrt[3]{3^{11}} \tau^{11}}{11 !}+\ldots
\end{aligned}
$$

As previously described, the imaginary terms of order $4 n+1, n=0,1,2, \ldots$ are eliminated by using the moment modification: $M^{a}(t, \tau)=e^{\text {jangle }(M(t, \tau))}$.

\section{THE GENERAL FORM OF THE DISTRIBUTION}

According to the previous analysis, the general form of the second order distribution can be defined. Namely, by observing the equations, (10), (18) and (21), which represents the individual distributions for the first, the second and the third order phase derivatives estimation, the unified two-terms moment function can be defined as follows:

$$
\begin{aligned}
M_{K}(t, \tau) & =\prod_{i=-1,}^{1} x^{\alpha(\sqrt{j})^{K}}\left(t+\frac{\sqrt[K]{K ! / 2} \tau}{\operatorname{sign}(i) \sqrt{j}}\right), \\
\alpha & = \begin{cases}\operatorname{sign}(i), \text { Kodd } \\
1, & K \text { even }\end{cases}
\end{aligned}
$$

where $K$ represent one of the considered phase derivatives. In order to decrease the spreading factor and to eliminate the influence of terms containing the imaginary unit $j$, the moment is firstly modified by using the exponential and angle functions as previously explained. Consequently, the general form of the complex-lag distribution is defined as:

$$
C T D_{N=2}^{K}(t, \omega)=\int_{-\infty}^{\infty} M^{a}(t, \tau) e^{-j \omega \tau^{K}} d \tau
$$

Hence, the same discretization points are used for all considered phase derivatives, while the scale factor and the exponent change according to the derivative order.

\section{EXPERIMENTAL RESULTS}

In this example, we consider a signal with fast-varying phase function given in the form:

$$
x=e^{\left.j(4 \cos 2 \pi t)+\frac{1}{3} \cos (3 \pi t)+\frac{2}{3} \cos (6 \pi t)\right)} .
$$

The proposed complex-lag distribution $C T D_{N=2}^{n}(t, \omega)$ for $n=1,2$ and 3 has been used to estimate and illustrate the first, second and third derivative of the phase.

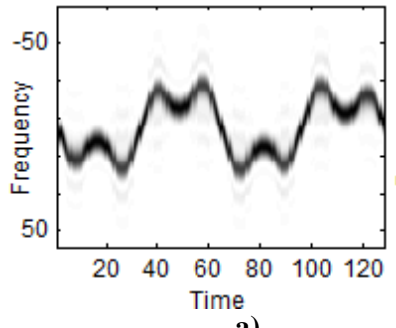

a)

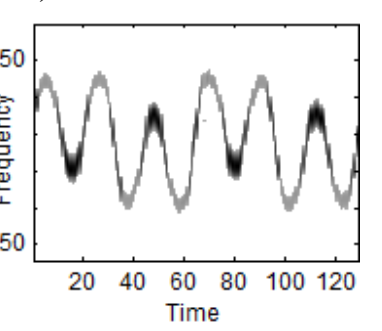

c)

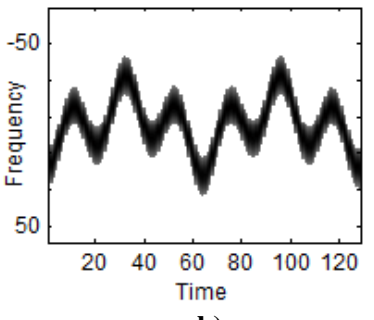

b)
Figure 1: The complex-lag distribution $C T D_{n}(t, \omega):$ a) $n=1$, b) $n=2$, c) $n=3$

According to the proposed approach, the modification of the moment based on the angle function is applied in all considered cases. The results obtained by the proposed distributions are shown in Figure 1.

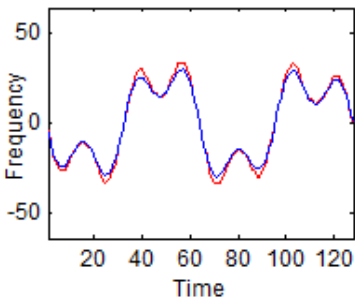

a)

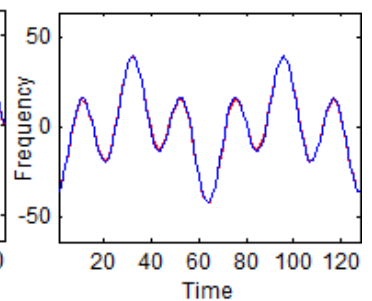

b)

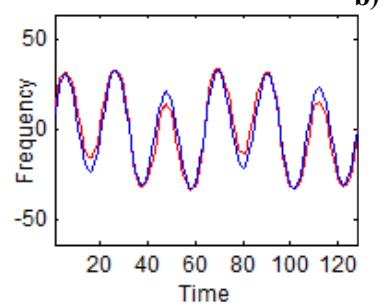

c)

Figure 2: The true (blue line) and estimated (red line): a) the first derivative of the phase, $b$ ) the second derivative of the phase c) the third derivative of the phase 
It may be observed that the considered signal is characterized by fast variations of signal phase function which is further reflected on the phase derivatives. However, even when the variations are fast, the proposed distribution provides an efficient representation of the considered phase derivatives, keeping the spreading factor still tolerable. Note that we have observed the nonnoisy signal. Namely, the estimation of higher phase derivatives is sensitive to noise, and thus the noisy signals should be filtered before the estimation procedure.

The true and estimated phase derivatives $(n=1,2$ and 3$)$ are shown in Figure 2. Mean Squared Error of the phase derivatives estimation are given in Table I. Note that the deviation of the estimated from the true values is low, especially when we have in mind that the variations of the estimated functions are very fast.

Table I: Mean Squared Errors of the phase derivatives estimations

\begin{tabular}{|l|c|}
\hline $\begin{array}{c}\text { Phase } \\
\text { Derivative }\end{array}$ & MSE \\
\hline $1^{\text {st }}$ & 4.4641 \\
\hline $2^{\text {nd }}$ & 1.1709 \\
\hline $3^{\text {rd }}$ & 28.5441 \\
\hline
\end{tabular}

\section{CONCLUSION}

A general and unified form of the distribution for the estimation of the first few phase derivatives (the first, the second and the third derivative) has been proposed. It is based on the second order signal moment (i.e., only two signal terms are employed). This general form has been derived from the specific distribution forms, obtained for each of the considered phase derivative estimation. The same pair of the discretization points on the unit circle is used for all three derivatives. The suitable points selection allows the use of the moment function modification that significantly reduces the spreading factor and increases the concentration along the estimated phase derivative. The proposed approach has been applied in the experiments with signals whose phase, as well as its derivatives, varies significantly even within a few samples. Although the phase variations were fast, the proposed distribution still provided good concentration for each of the considered phase derivatives.

\section{REFERENCES}

[1] L. Cohen, "Time-Frequency Distributions-A Review", Proceedings of the IEEE, Vol. 77, No. 7, July 1989.

[2] Proceedings of the IEEE, Special issue on Time-Frequency Analysis, vol. 84, no. 9, Sept. 1996.

[3] F. Hlawatsch and G. F. Boudreaux-Bartels, "Linear and quadratic time-frequency signal representations", IEEE Signal Processing Magazine, Vol. 9, No. 2, pp. 21-67, Apr. 1992.

[4] H. Choi, W. Williams, "Improved time-frequency representation of multicomponent signals using exponential kernels," IEEE Trans. on Acoustics and Speech, vol. ASSP37, no. 6, June 1989, pp. 862-871.

[5] M.G. Amin, W.J. Williams: "High Spectral Resolution Time-Frequency Distribution Kernels," IEEE Transactions on Signal Processing, vol. 46, no.10, Oct. 1998, pp. 27962804.

[6] E. Sejdić, I. Djurović, J. Jiang, "Time-frequency feature representation using energy concentration: An overview of recent advances," Digital Signal Processing, vol. 19, no. 1, pp. 153-183, January 2009.

[7] Y. Zhang, M. G. Amin, and G. J. Frazer, "High-resolution time-frequency distributions for manoeuvring target detection in over-the-horizon radars," IEE Proceedings on Radar, Sonar and Navigation, vol. 150, no. 4, pp. 299-304, Aug. 2003.

[8] P. O'Shea, "A New Technique for Instantaneous Frequency Rate Estimation", IEEE Signal Processing Letters, Vol. 9, No. 8, August 2002, pp. 251-252.

[9] S. Stanković, LJ. Stanković, "Introducing time-frequency distribution with a "complex-lag" argument", Electronics Letters, Volume 32, Issue 14, July 1996.

[10] S. Stanković, N. Zarić, I. Orović, C. Ioana, "General form of time-frequency distribution with complex-lag argument", Electronics Letters (ISSN:0013-5194), Volume 44, Issue 11, May 2008, Page(s) 699-701.

[11] S. Stanković, I. Orović, C. Ioana, "Effects of Cauchy Integral Formula Discretization on the Precision of IF Estimation: Unified Approach to Complex-lag Distribution and its L-Form", IEEE Signal Processing Letters, Vol. 16, No. 4, pp. 307-310, Apr. 2009.

[12] C. Cornu, S. Stanković, C. Ioana, A. Quinquis, LJ. Stanković, "Generalized Representation of Phase Derivatives for Regular Signals", IEEE Transactions on Signal Processing, Vol. 55, No. 10, pp. 4831-4838, September 2007. 